\title{
e-Migrinter
}

9| 2012

Immigrés, illégaux, réfugiés. Questions sur les enquêtes et les catégories

\section{Le morcellement de la catégorie statistique « réfugié »}

Luc Legoux

\section{OpenEdition}

\section{Journals}

Édition électronique

URL : https://journals.openedition.org/e-migrinter/770

DOI : 10.4000/e-migrinter.770

ISSN : 1961-9685

Éditeur

UMR 7301 - Migrinter

Édition imprimée

Date de publication : 20 juillet 2012

Pagination : 64-78

ISSN : 1961-9685

\section{Référence électronique}

Luc Legoux, "Le morcellement de la catégorie statistique « réfugié » », e-Migrinter [En ligne], 9 | 2012, mis en ligne le, consulté le 20 mai 2021. URL : http://journals.openedition.org/e-migrinter/770 ; DOI : https://doi.org/10.4000/e-migrinter.770 


\section{Le morcellement de la catégorie statistique « réfugié "}

\section{Luc Legoux}

Le morcellement de la catégorie "réfugié » a un rôle central dans la politique de réduction des flux de l'asile vers les pays occidentaux. La délocalisation de l'asile dans les pays d'origine est justifiée par la création de la catégorie "déplacés internes", catégorie qui recouvre aujourd'hui environ la moitié des personnes relevant du HCR (Haut Commissariat des Nations Unies pour les Réfugiés). La catégorie "protection subsidiaire », qui n’offre qu'une protection très limitée mais qui est comptée statistiquement avec les réfugiés statutaires, dissimule l'affaiblissement de la protection en Europe. Quant à la catégorie "demandeur d'asile », utilisée en France à partir de 1981 suite à des demandes frauduleuses de statut de réfugié, elle place tous les réfugiés sollicitant le statut en position de suspect. Ce morcellement de la catégorie « réfugié » résulte d'un changement du mode de gestion internationale de la protection des victimes de la violence qui reste inscrit dans la même logique d'assujettissement de l'asile aux politiques migratoires et étrangères à l'œuvre dès la création du HCR.

L'asile dans le monde (fin 2009)

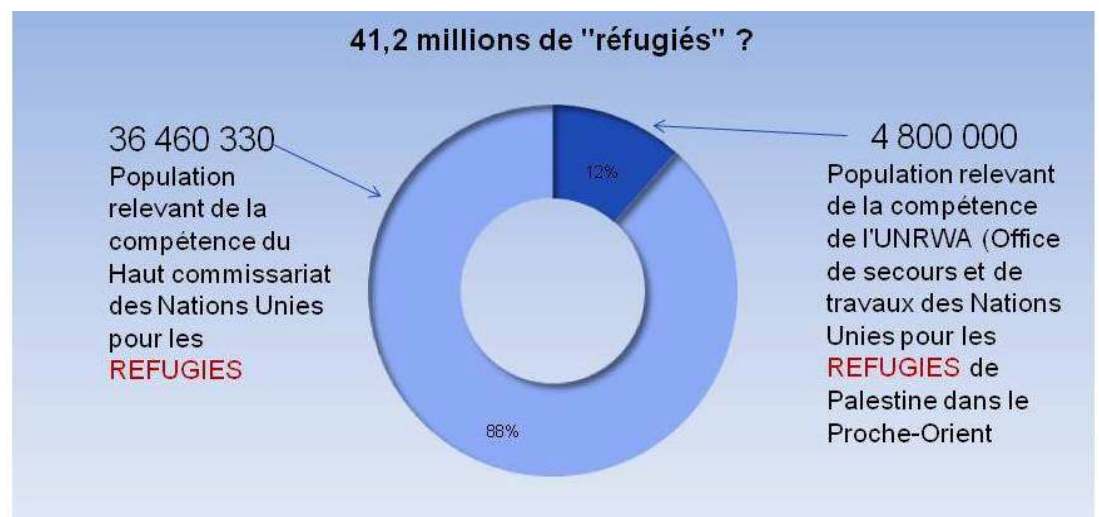

La séparation entre les personnes relevant du HCR et celle relevant de l'UNWRA (Office de secours et de travaux des Nations Unies pour les Réfugiés de Palestine dans le Proche Orient) a une origine historique. L'UNWRA a été créé en 1949 pour s'occuper spécialement des réfugiés de Palestine dans le Proche-Orient. Créé un an plus tard, essentiellement pour s'occuper des questions de réfugiés en Europe, le HCR a progressivement couvert toutes les autres situations de refuge sans remplacer l'UNWRA.

Quelle est le sens de l'addition du nombre de personnes relevant du HCR et de l'UNWRA ? C'est le nombre de personnes enregistrées comme étant en situation d'asile dans le monde. Ce ne sont pas tous des réfugiés puisque le rôle du HCR a été progressivement élargi à la protection d'autres catégories de personnes, ce n'est pas non plus le nombre total de personnes déplacées par les conflits ou les persécutions puisque l'enregistrement de ces personnes est loin d'être exhaustif. 


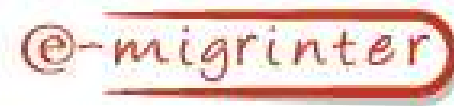

\section{Statistiques de l'UNHCR}

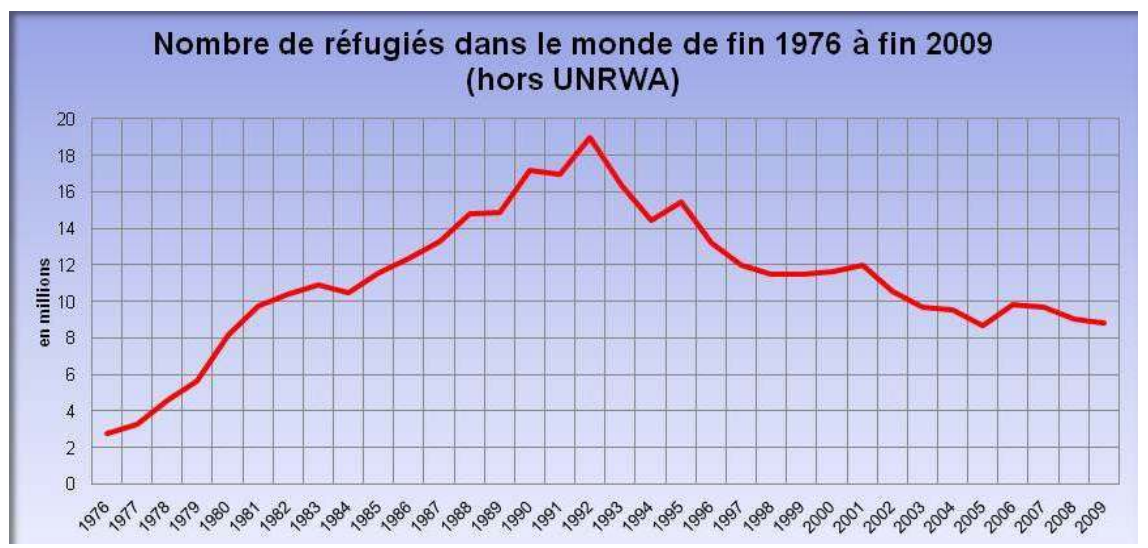

Le nombre de réfugiés dans le monde (hors UNWRA) est beaucoup plus faible, environ 9 millions en 2009. On constate sur ce graphique qu'il a diminué de moitié depuis 1993. Pourquoi cette baisse? Il ne s'agit malheureusement pas d'une amélioration de la situation des droits humains dans le monde mais d'un effet mécanique du morcellement de l'ensemble des personnes protégées en de multiples catégories.

Les 36,5 millions de personnes relevant du HCR fin 2009

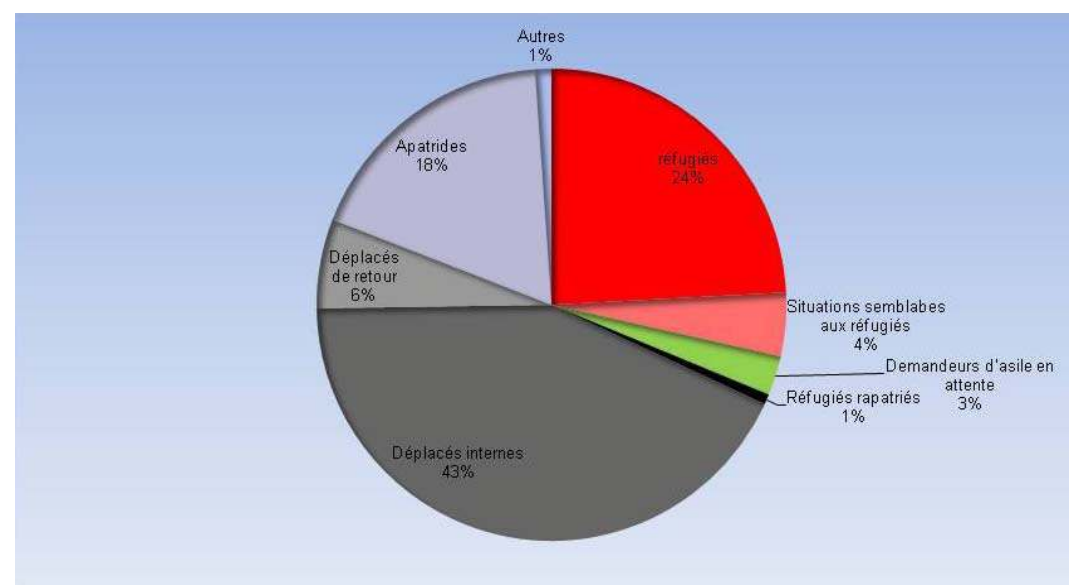

La répartition sur ce graphique des 36,5 millions de personnes relevant du HCR illustre ce morcellement. On constate d'emblée que les réfugiés sont marginalisés au sein du HCR. Les réfugiés reconnus comme tels ne représentent aujourd'hui qu'à peine un quart des personnes relevant du HCR alors que les personnes déplacées dans leur propre pays en représentent la moitié. Que signifie cette répartition? 


\section{Le morcellement est la conséquence d'un changement de mode de protection dans le cadre du système de Genève.}

L'évolution des catégories de l'asile n'est pas un changement de logique étatique de gestion de l'asile. C'est une adaptation du système de Genève au contexte politique et économique actuel.

\section{La logique de gestion de l'asile}

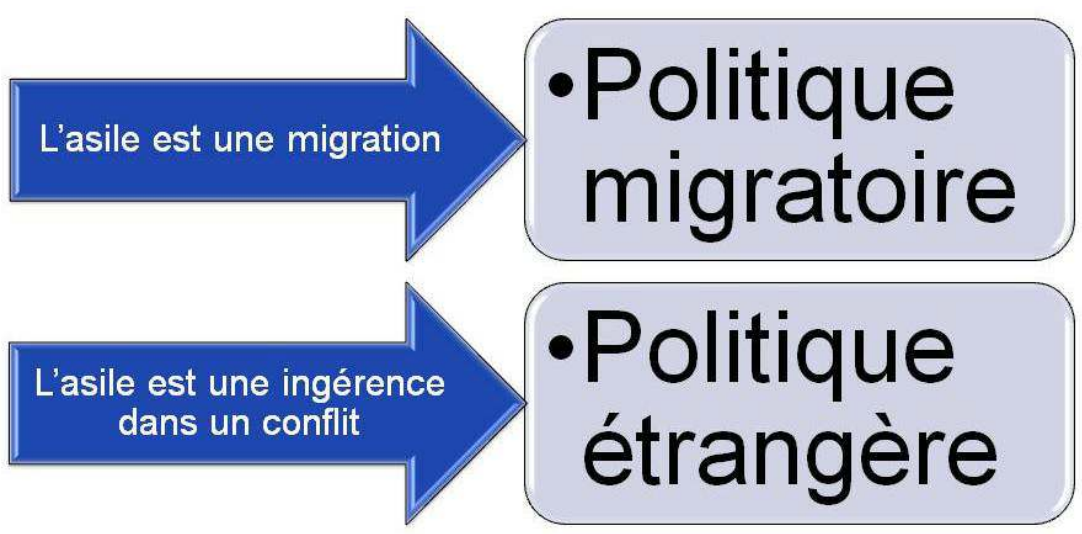

Pour les réfugiés, le système mis en place avec la Convention de Genève de 1951 relative aux réfugiés est un mode de protection. Pour les États d'accueil, ce système est un mode de gestion international des migrations de refuge par eux-mêmes et le HCR (rappelons que le HCR est financé par les États d'accueil sur la base du volontariat).

Ce mode de gestion doit adapter les modalités de la protection à la politique migratoire des pays d'accueil, puisque l'asile est une migration, et à leur politique étrangère puisque l'asile est une ingérence dans les conflits.

Contexte 1952

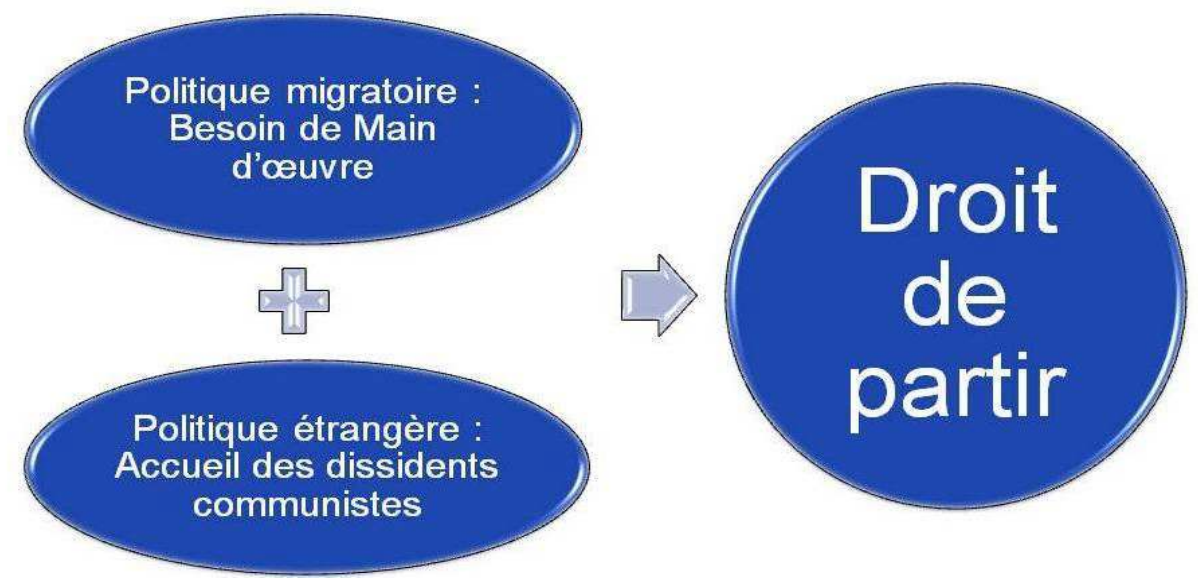

Aux débuts du système de Genève, les réfugiés arrivent essentiellement du monde communiste, en petit nombre du fait de la fermeture de ces pays. Sur le plan politique, leur 
accueil permet de souligner le caractère persécuteur du régime communiste. Sur le plan économique, le besoin de main-d'œuvre impose un recours à l'immigration. Le mode de protection des réfugiés est donc basé sur leur droit de partir de leur pays d'origine et d'être accueillis en Occident.

\section{Contexte 1989}
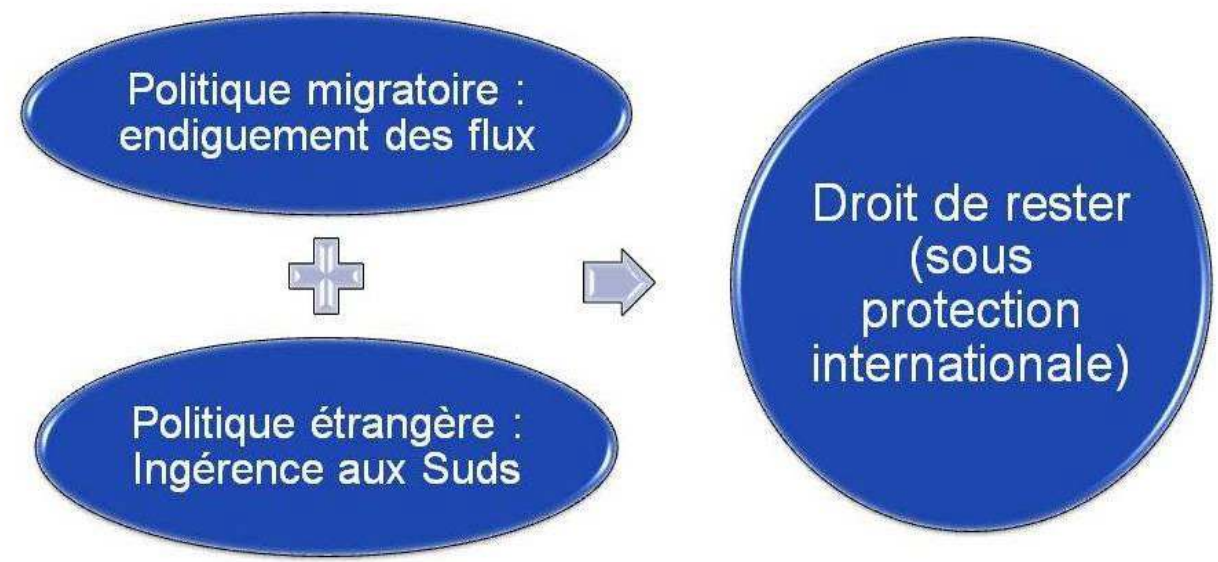

Avec la fin de la guerre froide, l'intérêt politique d'accueillir les réfugiés en occident ou dans les camps dans les régions d'origine disparait. À l'inverse, l'heure est à l'ingérence dans les conflits aux Suds. Avec la mondialisation et la montée du chômage, depuis le milieu des années 1970, la politique migratoire des pays riches est devenue très restrictive. Pour limiter les flux migratoires vers les pays riches, un nouveau mode de protection des victimes des conflits basé sur le droit de rester en sécurité dans son propre pays est progressivement mis en place.

\section{La création de la catégorie «déplacés internes»}

Un changement de mode de protection ... Mais pas de logique de gestion de l'asile

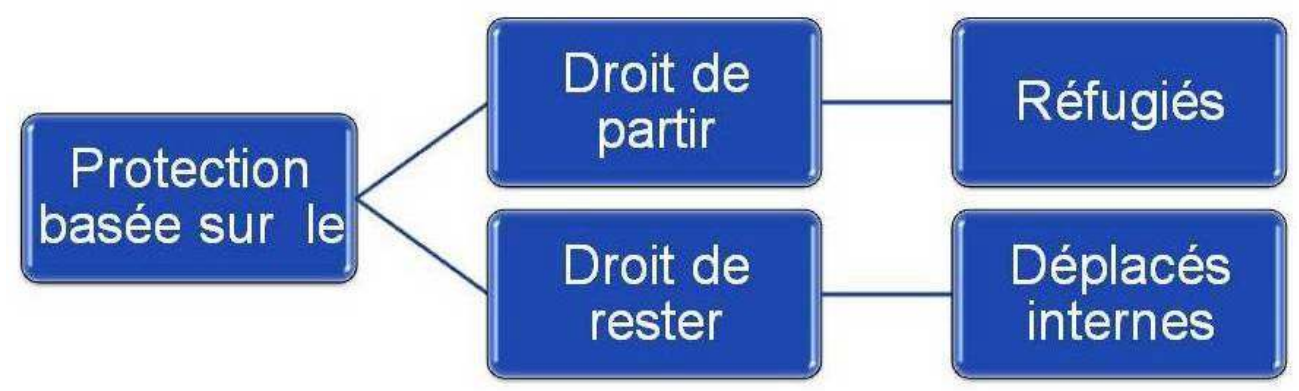

La création de la catégorie " personnes déplacées » répond à cette évolution. Les catégories statistiques de l'asile correspondent aux modes de protections choisis selon les relations internationales et les politiques migratoires du moment. 
Le droit de partir implique une catégorie «réfugiés statutaires » comme le droit de rester implique une catégorie "personnes déplacées ». Le mode de protection a changé, mais pas la logique de gestion de l'asile.

\section{La création de la catégorie « demandeur d'asile »}

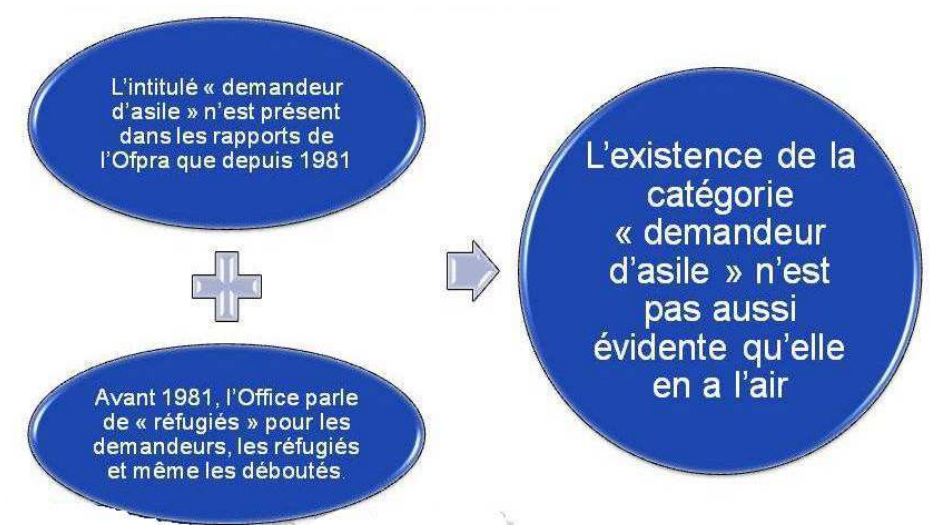

La catégorie « demandeur d'asile » est d'un usage si courant aujourd'hui qu'elle semble être aussi ancienne que la catégorie "réfugié » elle-même. C'est une fausse impression car l'usage officiel de cette catégorie est en fait très récent. On ne trouve aucune trace de cette catégorie dans les rapports d'activité de l'Office français de protection des réfugiés et apatrides (Ofpra) avant 1981, ce qui signifie que durant toute la première moitié de l'existence de la Convention de Genève (qui a eu 60 ans en 2011), cette catégorie n'était pas employée par l'Office chargé de reconnaitre la qualité de réfugié.

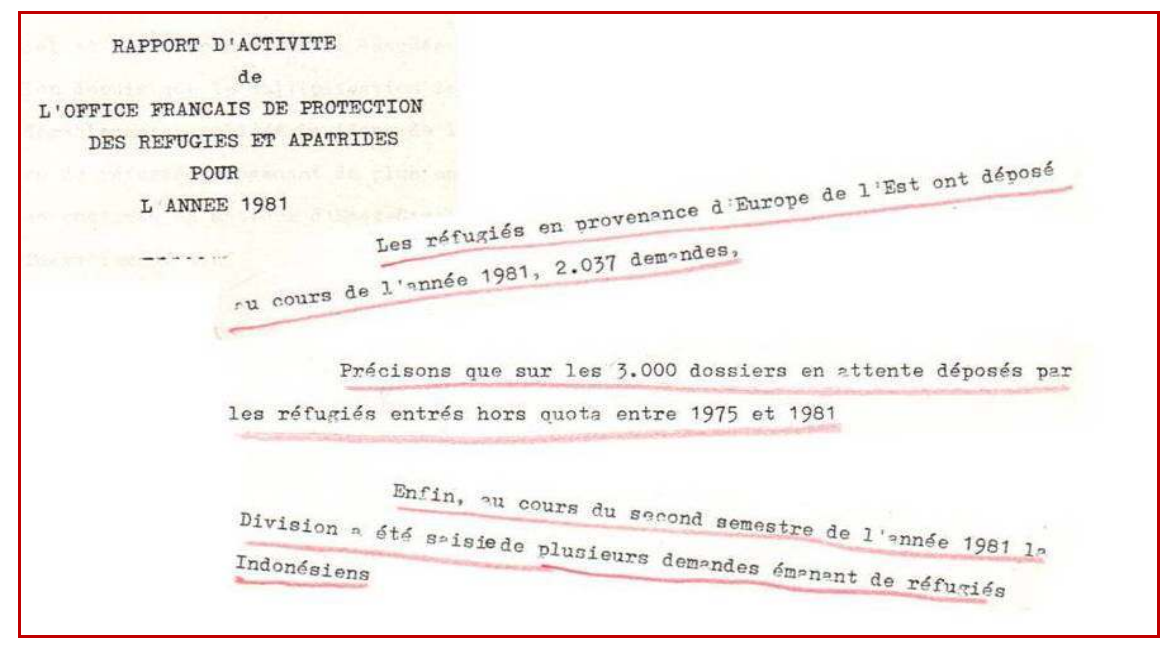

Comme le montrent les citations présentées ci-dessus, avant 1981, l’Ofpra employait le mot « réfugié » pour désigner ceux que l'on nomme aujourd'hui les « demandeurs d'asile ». Écrire que des réfugiés ont déposé des demandes, formulation inconcevable aujourd'hui, signifie, qu'à l'époque, les personnes en attente de statut étaient considérées comme des réfugiés jusqu'à preuve du contraire. 
RAPPORT SUR L'ACTIVINE DE L'OFFICE FRANCAIS

DE PROTECTION DES REFUGIES ET APATRIDES

PENDANT LA PERIODE COMPRISE. ENTRE

LE 22 SEPTE! BRE 1952 et le 31 DECBMBRE 1953

$== \pm===x=\pi$

Les réfugiés à qui l'office a refusé de reconnâtre cette qualité sont habilités à adresser des requêtes à la Commisdans les délais fixés, à l'effet de solliciter l'examen de

leurs recours.

Même en cas de refus de reconnaissance de la qualité de réfugié par l'Office, ceux que l'on nomme aujourd'hui les déboutés étaient encore nommés réfugiés tant qu'ils disposaient d'un droit de recours. La présomption de bonne foi ne disparaissait qu'après la confirmation du rejet par la Commission des recours des réfugiés (renommée aujourd'hui Cour nationale du droit d'asile, CNDA).

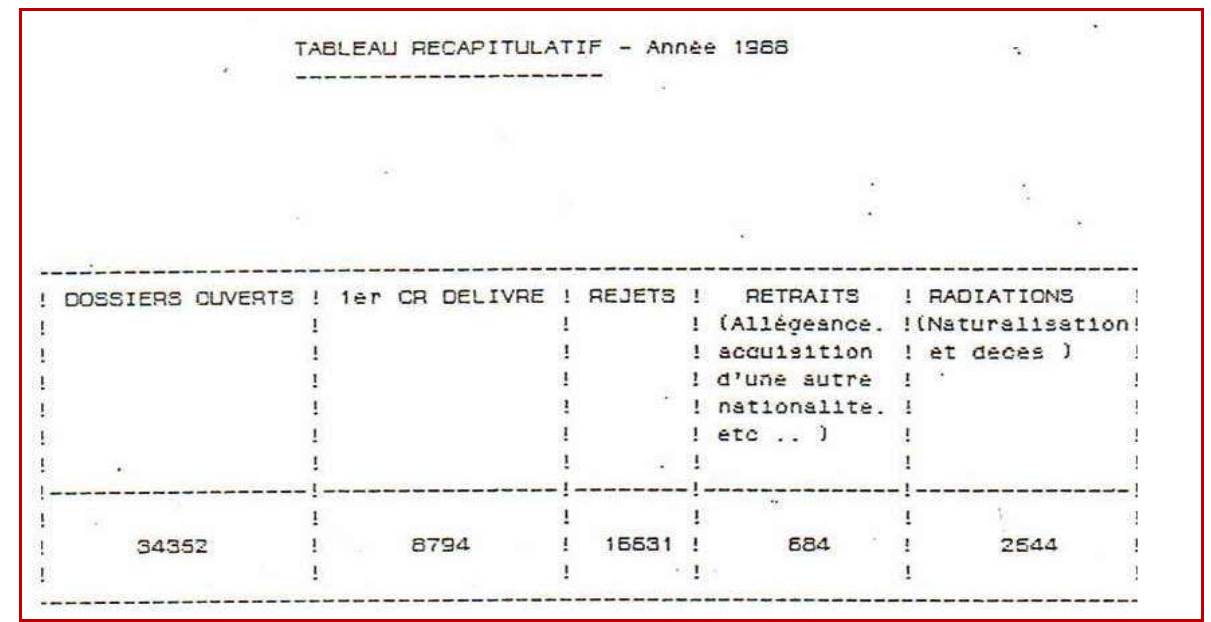

Dans les tableaux statistiques, l'Office utilisait l'expression «dossiers ouverts » et l'a conservée jusqu'en 1988. On peut en déduire qu'au moins 7 ans ont été nécessaires pour la généralisation de l'usage de la catégorie « demandeur d'asile ». 


\section{Le statut de réfugié est recognitif}

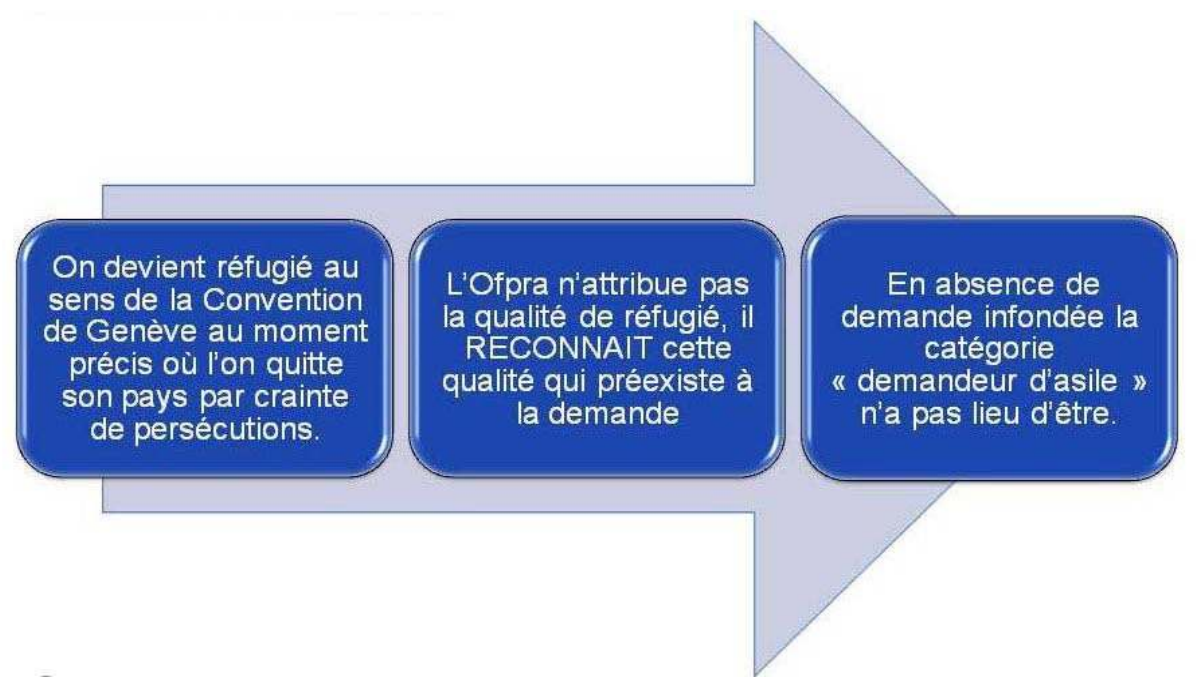

Le fait d'utiliser le mot "réfugié » pour désigner les personnes en attente de la reconnaissance de leur statut de réfugié jusqu'en 1981 n'est pas un manque de vocabulaire, c'est une conséquence du concept même de réfugié.

Le statut est recognitif, ce qui signifie que l'Ofpra reconnait la qualité de réfugié des personnes qui sont devenues réfugiées en quittant leur pays par crainte de persécutions. Lorsque toutes les demandes sont fondées sur des persécutions, tous les demandeurs sont déjà réfugiés de fait avant d'être reconnus comme tels en droit. C'est l'existence de demandes frauduleuses, infondées, ou jugées infondées qui conduit à créer une catégorie distincte pour les demandeurs d'asile.

\section{Passage du réfugié au suspect}

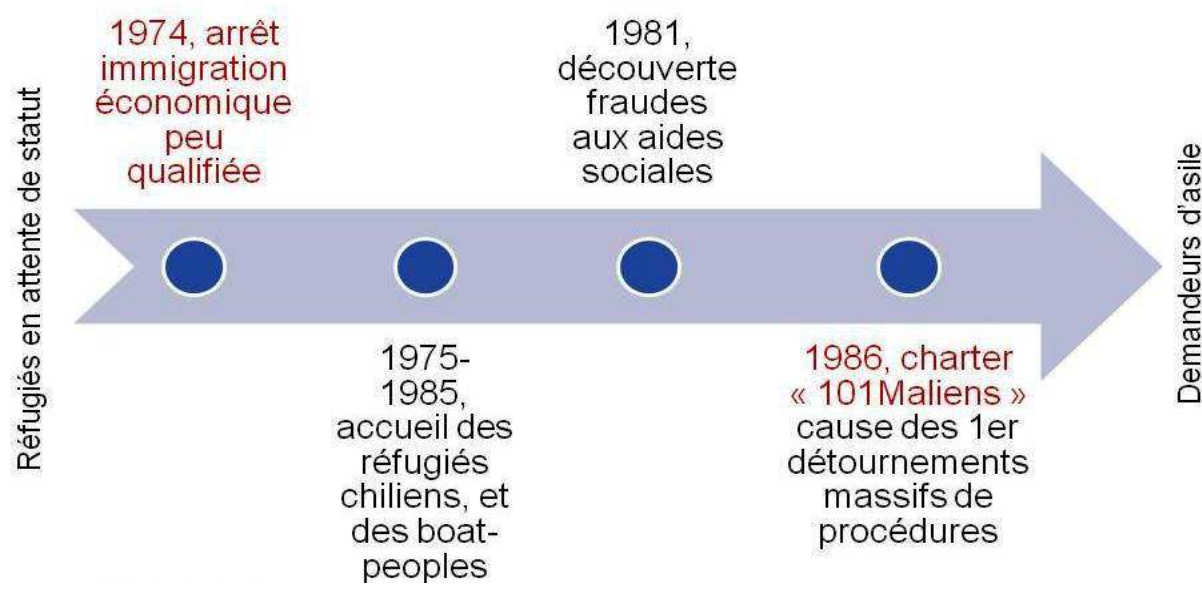

La chronologie indiquée ci-dessus montre le passage du réfugié en attente de statut, présumé de bonne foi, au demandeur d'asile suspecté d'être un immigré économique utilisant la procédure d'asile pour obtenir un droit au séjour. 
Les premiers détournements massifs sont constatés après l'opération très médiatisée de renvoi par charter de 101 maliens immigrés illégaux. Ce ne sont pas des arrivées récentes en France mais un phénomène de vases communicants entre illégalité et demande d'asile.

\section{Rapport Ofpra de 1986}

Par ailleurs, les mesures prises en France, comme dans d'autres pays occidentaux, pour mieux contrôler les migrations à motivation économique semblent avoir eu des effets contradictoires sur le nombre des personnes qui se sont adressées à l'O.F.P.R.A. : si d'éventuels candidats au statut de réfugié ont pu renoncer à venir en France ou se voir interdire l'accès du territoire national, en revanche, bien des immigrés en situation plus ou moins irrégulière ont probablement été tentés de recourir aux procédures de l'asile politique pour écarter la menace de mesures d'expulsion. Ce n'est sans doute pas une simple coīncidence si le nombre des requérants maliens qui ne dépassait pas en moyenne 10 par mois en 1985 a atteint 34 en octobre, 43 en novembre et 52 en décembre 1986.

La parfaite connaissance officielle de ce phénomène de vases communicants, illustrée par cette citation d'un rapport d'activité de l'Ofpra qui fait référence à l'expulsion par charter des 101 maliens organisé par le ministre de l'Intérieur Charles Pasqua, est peu médiatisée. Au contraire, les demandes d'asile non fondées sont toujours présentées comme un moyen d'entrer en France alors que c'est surtout un moyen d'obtenir un titre de séjour temporaire pour ceux qui sont déjà en France.

\section{L'extension de la catégorie « réfugié »}

Réfugiés conventionnels : définition jusqu'en 1994

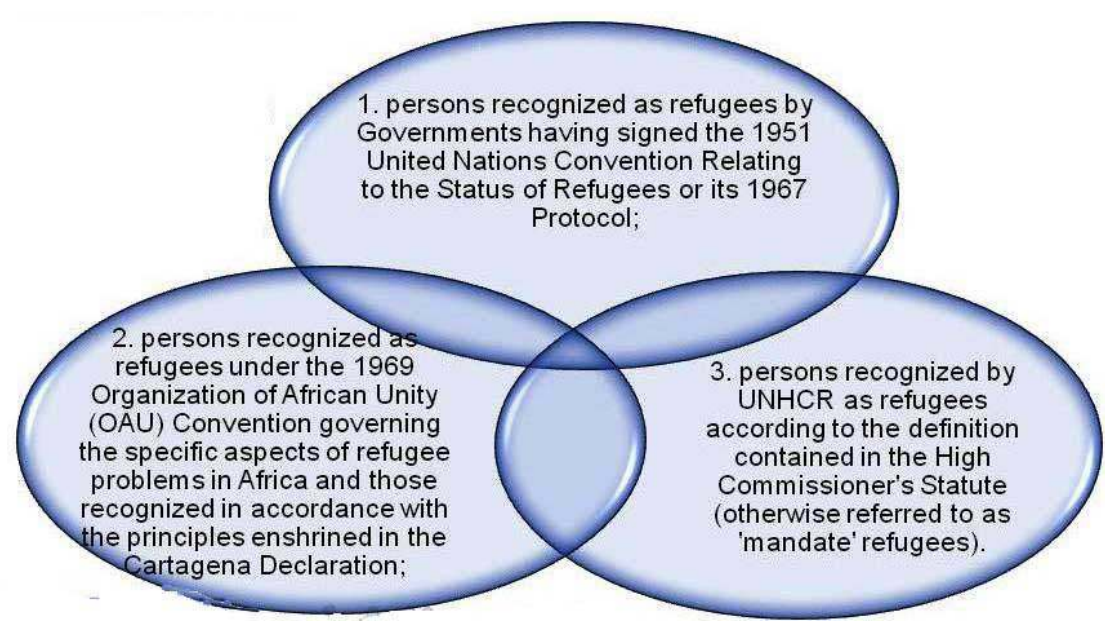

La définition de la catégorie statistique "réfugié », limitée à l'origine aux réfugiés reconnus au titre de la Convention de Genève et du mandat du HCR, a inclus les réfugiés reconnus au titre 
de la Convention de l'OUA et de la Déclaration de Carthagène à partir de leur entrée en vigueur. C'est deux textes reprennent la définition de la Convention de Genève et l'élargissent aux victimes de guerres ou de violences généralisées.

Dans un apparent paradoxe, le morcellement de la catégorie statistique réfugié s'accompagne, à partir de 1994, d'une extension de la définition de ceux qui sont comptabilisés comme réfugiés dans les statistiques.

1995

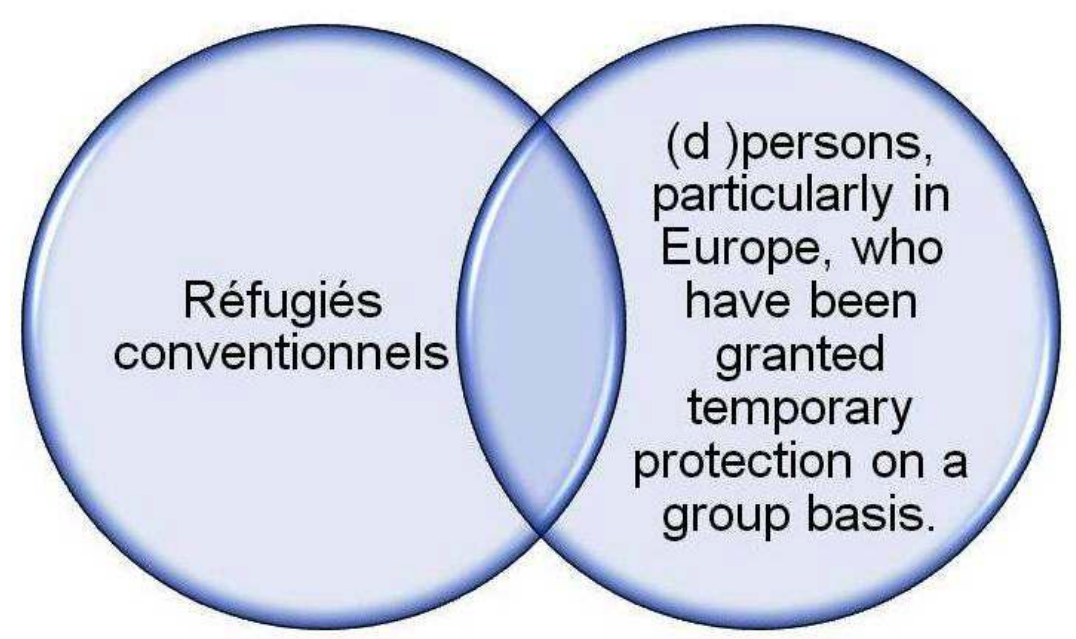

En 1995, des personnes n'ayant obtenu qu'une protection temporaire notamment en Europe, c'est-à-dire ne bénéficiant pas de la protection du statut de réfugié, sont intégrées dans la catégorie statistique « réfugié ».

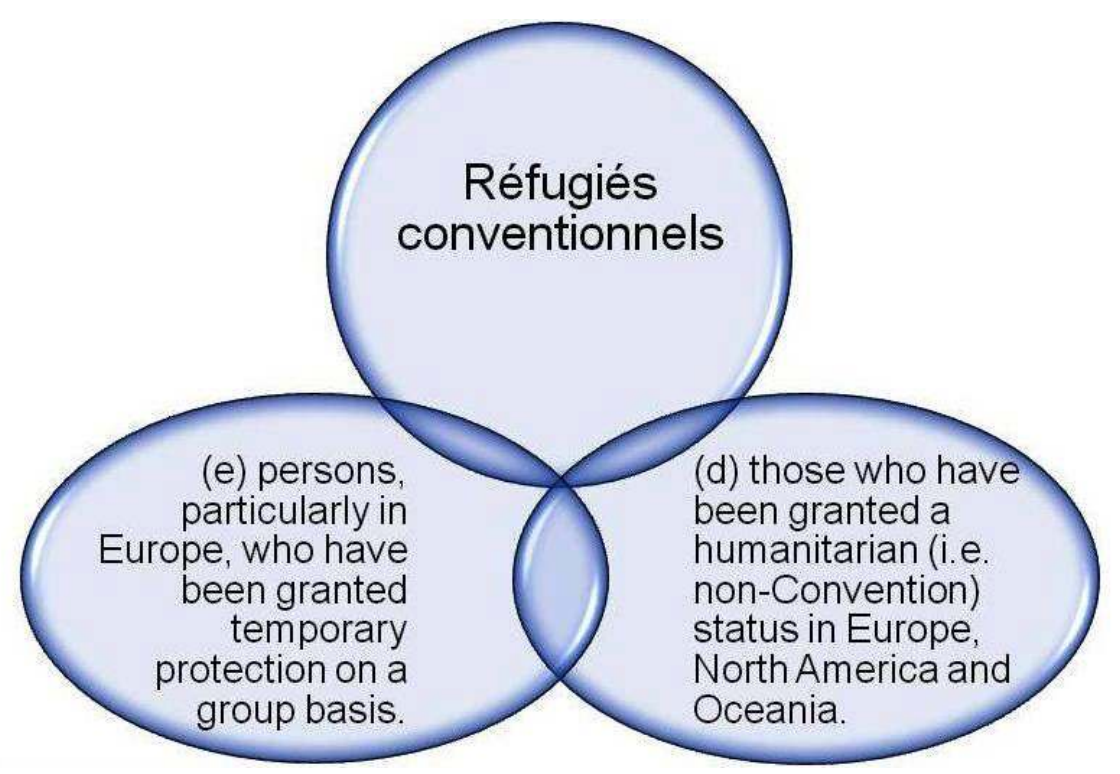

Les divers sous-statuts humanitaires inventés par les pays d'accueil pour gérer les populations auxquelles ils ne veulent pas reconnaitre le statut de réfugié, mais qu'ils sont dans 


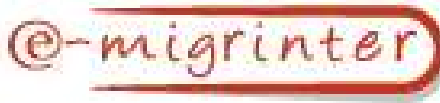

l'impossibilité d'expulser du fait des circonstances dans leurs pays d'origine, sont ajoutés dans la catégorie statistique « réfugié » en 1996.

\section{Formulation 2009}

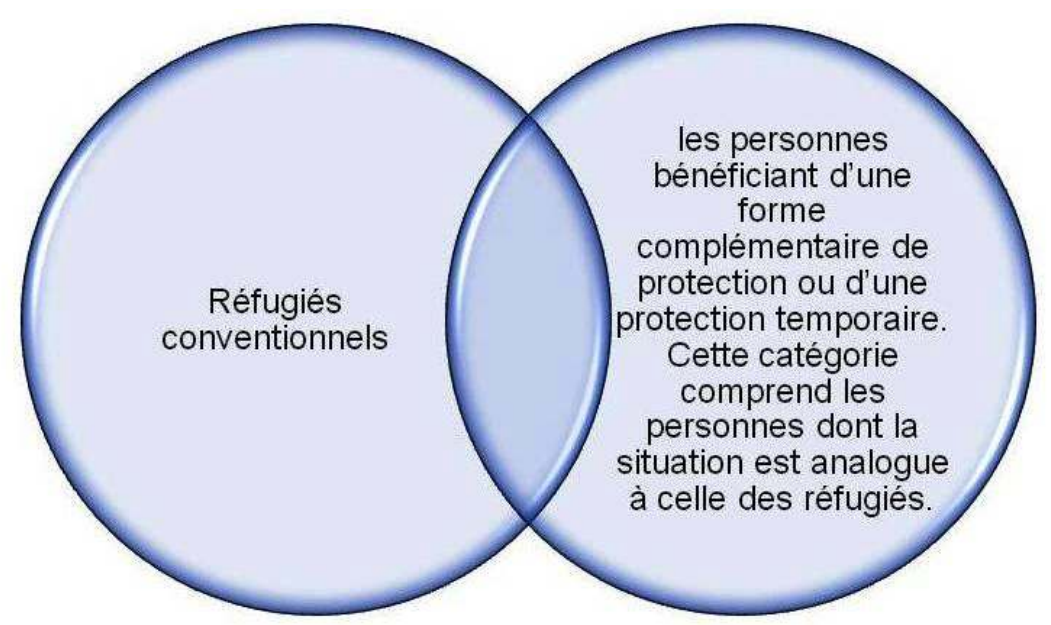

À partir de 2009, la catégorie statistique « réfugié » englobe même des personnes sans le moindre statut mais dont la situation est analogue à celle des réfugiés.

Sens de l'extension de la catégorie statistique « réfugié »

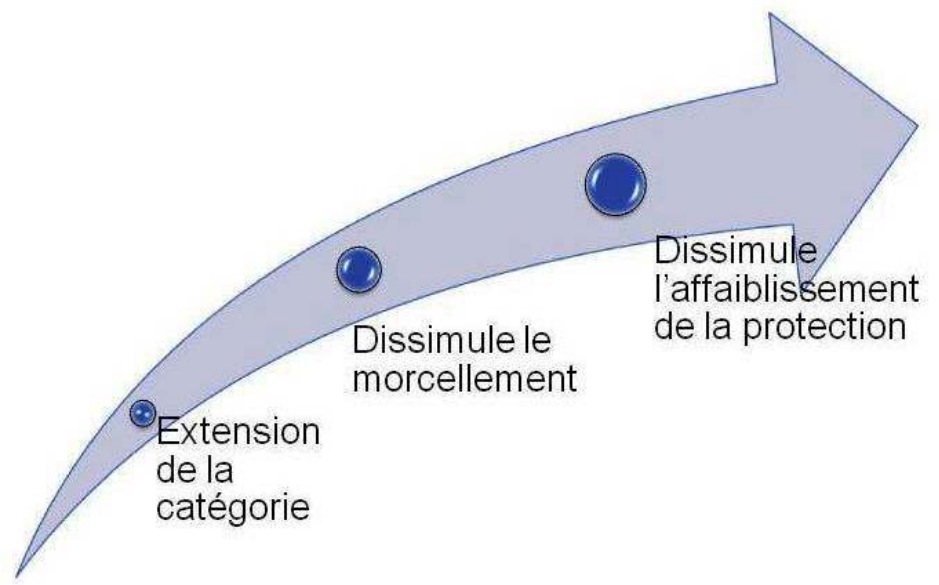

Le paradoxe entre le morcellement des statuts et l'extension de la catégorie statistique n'est qu'apparent puisque les deux phénomènes poursuivent le même but : réduire les flux migratoires vers l'Europe. La protection sur place dans les pays d'origine vise à tarir les flux à la source et l'extension de la catégorie statistique cherche à dissimuler la baisse de la qualité de la protection dans les pays qui ne souhaitent pas abandonner leur image de patries des droits humains. 
L'importance de la protection complémentaire : reconnaissances 2009 Union européenne

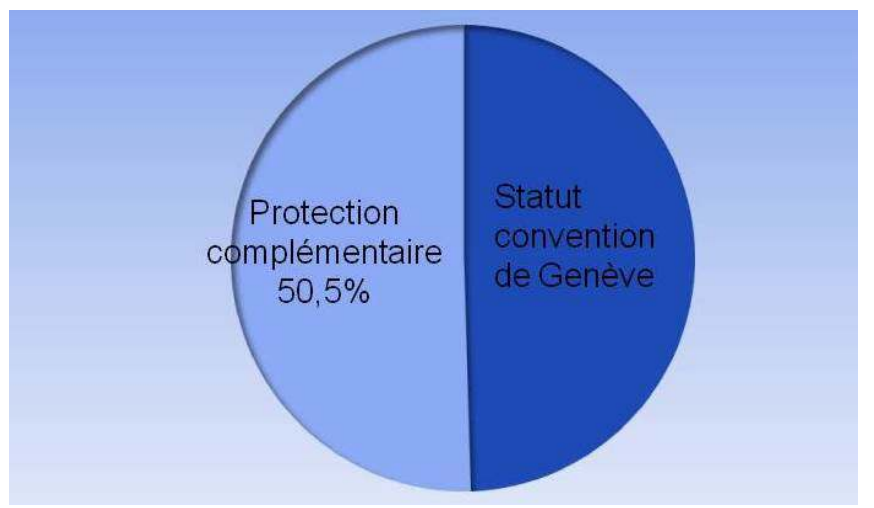

Dans l'Union européenne, plus de la moitié des personnes dites « réfugiées » n'ont pas le statut de réfugié de la Convention de Genève mais un sous-statut nommé "protection subsidiaire ». Selon les textes, «Le bénéfice de la protection subsidiaire est accordé à toute personne qui ne remplit pas les conditions pour se voir reconnaittre la qualité de réfugié et qui établit qu'elle est exposée dans son pays à l'une des menaces graves suivantes : a) La peine de mort ; b) La torture ou des peines ou traitements inhumains ou dégradants ; c) S'agissant d'un civil, une menace grave, directe et individuelle contre sa vie ou sa personne en raison d'une violence généralisée résultant d'une situation de conflit armé interne ou international. »

Pour ne pas remplir les conditions de reconnaissance de la qualité de réfugié, il faut que les motifs de persécutions ne soient liés ni à la race, ni à la nationalité, ni à la religion, ni à l'appartenance à un certain groupe social, ni aux opinions politiques. La notion de groupe social étant extensible, bien peu de cas ne peuvent réellement pas entrer dans les critères de la Convention de Genève. L'important usage de la protection subsidiaire ne s'explique que par la volonté de réduire la qualité de la protection accordée.

Différence entre les types de protections (en France)
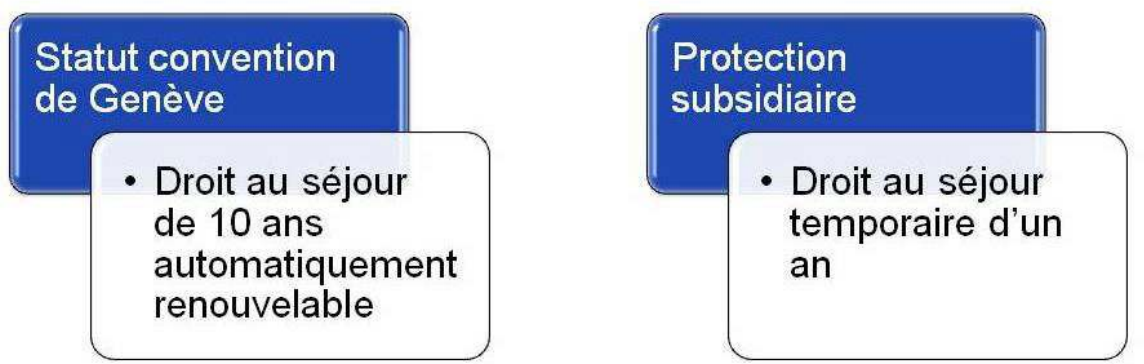

Comme le montre le droit au séjour associé, 1 an au lieu de 10 pour le statut de réfugié, la protection subsidiaire est réellement un sous-statut en termes de protection. Même lorsqu'elle dure de nombreuses années, la protection subsidiaire rend les conditions d'une intégration dans le pays d'accueil très difficile. 


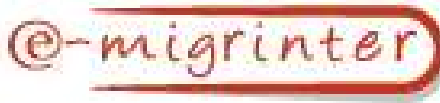

\section{La protection subsidiaire en France : \% de l'ensemble des personnes protégées}

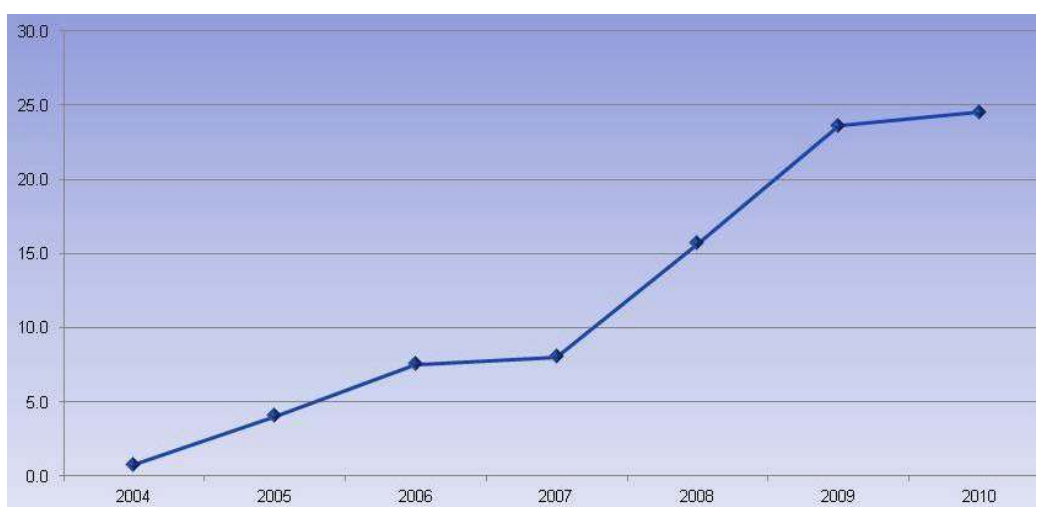

En France, la protection subsidiaire s'est substituée à l'asile territorial. Conçue pour protéger les victimes de persécutions perpétrées par des groupes armés non étatiques, et relevant à l'époque du ministère de l'Intérieur, cette forme de protection n'était que très peu accordée. Depuis 2004, l'usage de ce sous-statut s'accroit régulièrement.

\section{Diminution du poids des réfugiés : la révision statistique de 2006}

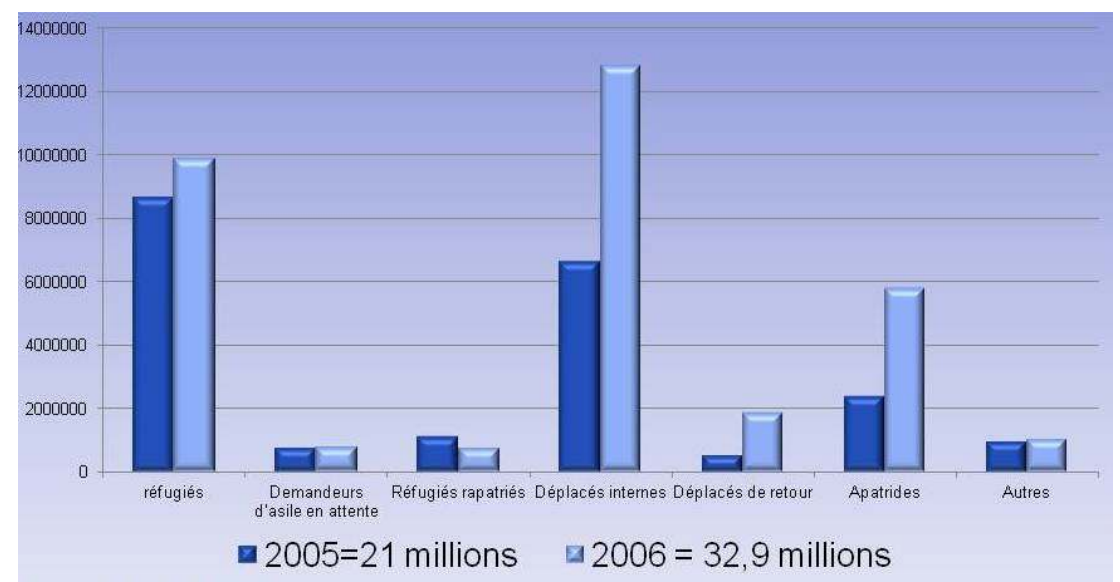

Les statistiques du HCR sont issues de données gouvernementales ou d'estimations lorsque les données sont incomplètes. L'interprétation de l'évolution des effectifs doit tenir compte de cette fragilité. C'est ainsi que la brutale augmentation de plus de 50\% entre 2005 et 2006 n'est pas liée à un accroissement des conflits dans le monde mais à une révision de l'estimation du nombre des personnes déplacées et des apatrides, auparavant sous-estimé.

En 2006, l'estimation du nombre de réfugiés, notamment dans les pays anglo-saxons, a également été revue à la hausse, mais dans des proportions moindres, et le poids des réfugiés dans l'ensemble a donc diminué. Rappelons également que le HCR est financé sur contribution volontaire des États, et que les statistiques sont un moyen de communication. 


\section{Révision des estimations entre 2005 et 2006 (exemples)}
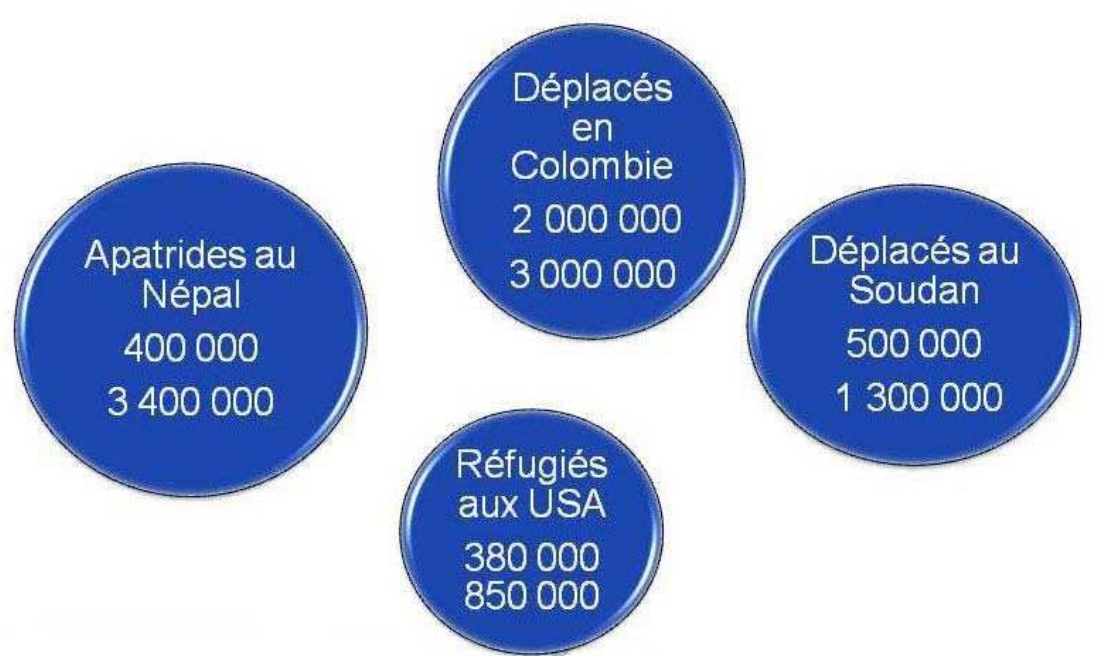

Voici quelques exemples de l'importance des révisions du nombre de personnes déplacées et d'apatrides. La croissance du nombre de réfugiés aux USA est le résultat d'un changement de mode de calcul. En effet, les États-Unis ne connaissant pas le nombre de réfugiés sur leur territoire, le HCR l'estime par la somme des délivrances de statut des 5 dernières années jusqu'en 2005 et par la somme des 10 dernières années depuis 2006.

Les catégories « invisibles »

\section{Les dublins : en France, 5943 saisines en 2009 soit l'équivalent de $16,1 \%$ du nombre des premières demandes d'asile.}

\section{Les déboutés : en France, 32571 en 2010}

Les dublins sont les personnes sous procédure de renvoi dans un pays membre de l'Union européenne (règlement Dublin 2). Les déboutés sont à la marge de l'État de droit en France, le système juridique sait refuser de reconnaitre la qualité de réfugié dans le respect formel du droit, mais la réalité des situations concrètes s'oppose souvent à la réussite des décisions d'éloignement du territoire, seul environ $20 \%$ de ces décisions sont exécutées. Les déboutés ne restent pas pour autant éternellement en France, la dureté de la situation d'illégal les incite à tenter leur chance ailleurs. 


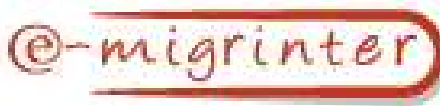

Une catégorie en devenir : « les déplacés catastrophes naturelles »

\begin{tabular}{|l|r|}
\hline Category of forced displacement & Total (in $\mathrm{mln}$ ) \\
\hline Refugees under UNHCR mandate & 11.4 \\
Refugees under UNRWA mandate & 4.6 \\
\hline Total number of refugees & 16.0 \\
\hline Conflict-generated IDPS & 26.0 \\
Natural disaster IDPS & 25.0 \\
\hline Total number of IDPs & 51.0 \\
\hline Total number of refugees and IDPS & 67.0 \\
\hline
\end{tabular}

Source : HCR, Tendances mondiales en 2007.

Le HCR n'est pas favorable à l'appellation «réfugié » pour les victimes des catastrophes naturels. La tentation de les intégrer dans son champ de compétence existe cependant puisque dans le rapport "Global trends » de 2007 les IDPs (déplacés internes) pour cause de désastre naturel sont inclus dans le total des réfugiés et déplacés internes.

Conclusion :

Le rôle central du morcellement et du nom des catégories

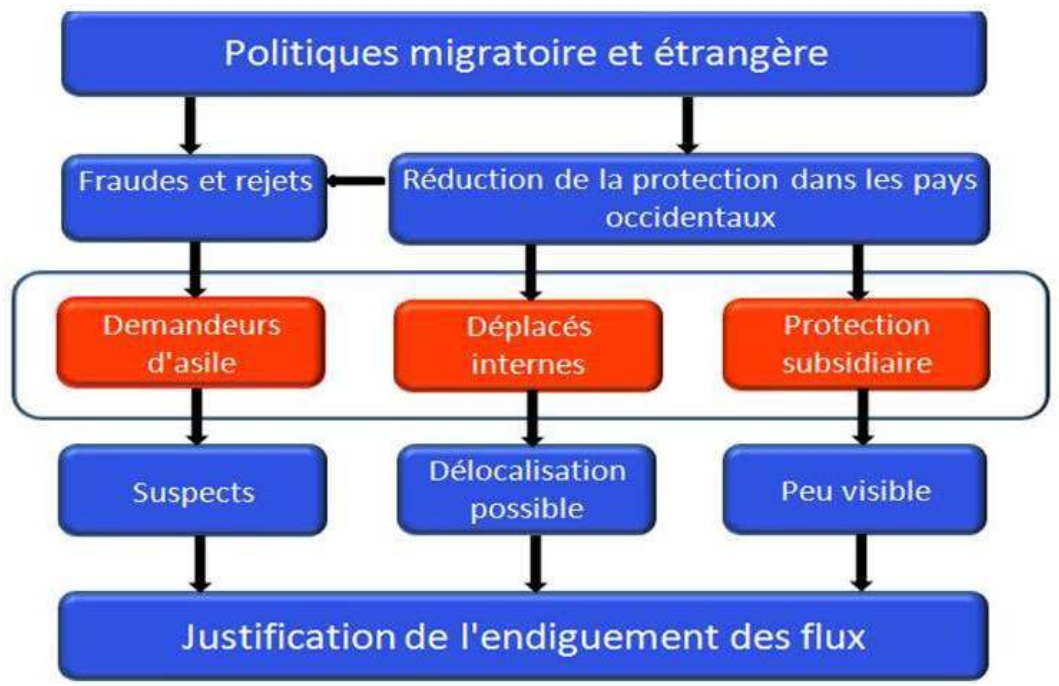

Les politiques migratoires produisent les conditions de leur propre justification. En réduisant la protection dans les pays occidentaux, elles induisent d'une part la création des catégories « déplacés internes » et «protection subsidiaire », et d'autre part des détournements de procédures qui induisent la catégorie « demandeurs d'asile». 
Les demandeurs d'asile sont des suspects, les déplacés internes montrent que la délocalisation de l'asile est possible, l'expression "protection subsidiaire » qui remplace celle de «sous-statut » dissimule la baisse de la protection en Union européenne. Le rôle des expressions «demandeurs d'asile» et "protection subsidiaire» nous rappelle une fois de plus que les mots sont les armes de la politique.

Luc Legoux

MCF en Démographie

Institut de Démographie de l'Université de Paris 1 (IDUP)

Migrinter - UMR 7301

luc.legoux@univ-paris1.fr 